\title{
5. ELECTRICAL ACTIVITY OF THE OLFACTORY BULB AS MODIFIED BY HYPOTHALAMUS AND RETICULAR FORMATION STIMULATION
}

\author{
Chosaburo Yamamoto, M.D. AND Kitsuya IWAma, M.D. \\ Department of Physiology, Medical School, University of Kanazawa, Kanazawa
}

In recent years, it has been well recognized that the afferent conduction for sensory perception is put under control of the higher centers. Concerning the olfactory system, the first to deal with this problem were Arduini and Moruzzi ${ }^{1}$, who attempted to modify electrical activity of the olfactory bulb by high frequency stimulation of the mesencephalic reticular formation (RF). They, however, failed to find any noticeable effects of RF stimulation upon activity of the olfactory bulb. Later, Kerr and Hagbarth ${ }^{2}$ found that repetitive stimulation of the anterior limb of the anterior commissure suppressed activity of the olfactory bulb and considered that this phenomenon might be due to activation of the centrifugal fibers entering the olfactory bulb. However, as the anterior limb of the anterior commissure contains the centrifugal as well as the centripetal fibers ${ }^{3)}$, a question remains to be answered to what extent antidromic stimulation of the centripetal fibers is involved in the phenomenon as described by Kerr and Hagbarth.

The present experiment was designed to study regulatory influences of the subcortical activating system, including the RF and the hypothalamus, upon activity of the olfactory bulb. The results clearly showed that the latter received remarkable effects from the subcortical activating system.

\section{Method}

The experiments were carried out on curarized rabbits maintained on artificial respiration. Recordings of the electrical activity were made by bipolar silver electrodes placed gently on the dural surface of the olfactory bulb. Deep stimulation was carried out by using a concentric bipolar electrode introduced stereotaxically. For olfactory stimulation a polyethylene cannula was inserted into the postnasal cavity through the oral end of the transected trachea and was connected to the inlet pipe of an artificial respirator, which periodically admitted room air through the nose.

In all experiments, the sites of the stimulating electrodes were determined histologically, in frozen sections stained with haematoxylin. 


\section{Results}

With the olfactory bulb of the unanaesthetized rabbits, two types of the potential wave have been described. The first, the intrinsic wave, is recorded in the absence of the olfactory stimulus and has a frequency of about $100 \mathrm{cps}$. This wave can be observed even if the olfactory bulb is isolated neuronally from the central nervous system and from the olfactory mucosa. The second type, the induced wave, is an oscillatory activity caused by olfactory stimulation. The frequency of this wave is $30-50 \mathrm{cps}$ and usually the amplitude is conspicuously larger than that of the intrinsic wave.

$R F$ stimulation: High frequency stimulation of the RF produces the arousal pattern in EEG of the neocortex; spindle bursts and slow waves in EEG are replaced with low voltage fast activity. Concerning the olfactory bulb, repetitive stimulation in the RF suppressed the induced and the intrinsic waves. In Fig. 1, A, room air was introduced through the nostril at a frequency of $0.3 \mathrm{cps}$ and the induced wave was brought about separately in response to each olfactory stimulus. By RF stimulation (underlined), the induced wave was suppressed profoundly, and this suppressing effect continued for several seconds after the stimulus was turned off. At the same time as RF stimulation began, the intrinsic wave was also decreased in amplitude (Fig. 1. B-2). In this case the original rhythm of about $100 \mathrm{cps}$ disappeared almost completely leaving irregular slow oscilla-

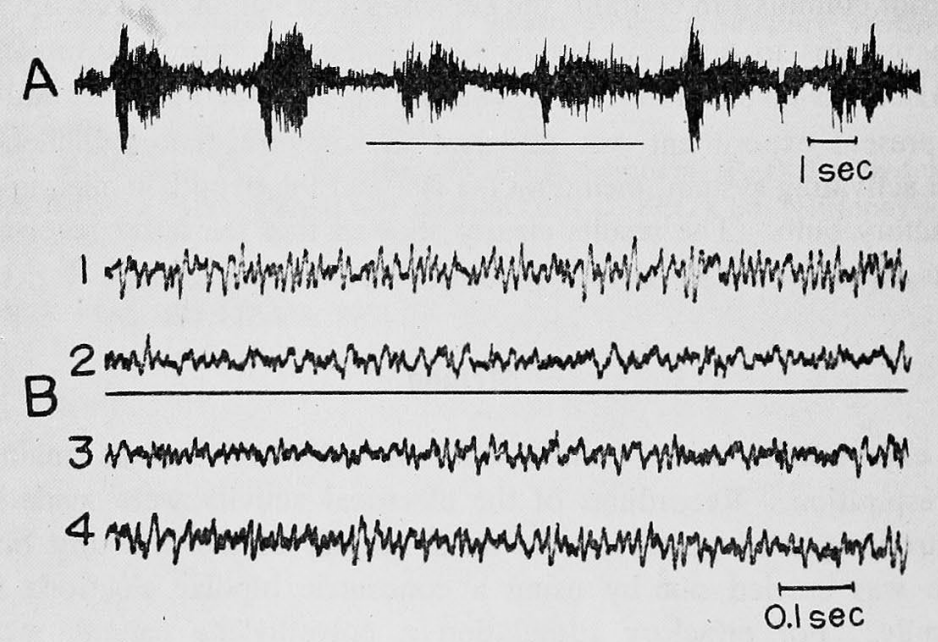

Fig. 1. Effects of RF stimulation. Solid lines indicate RF stimulation. $A$; induced wave was suppressed through and after stimulation.

B; intrinsic wave. Rhythmic oscillation at pre-stimulation period

(1) was suppressed immediately after the commencement of stimulus (2). Stimulation was continued for about $3 \mathrm{sec}$. $3 \& 4 ; 1$ and $4 \mathrm{sec}$ after turning off stimulus. 
tions. Similar suppressing effects were obtained by stimulating the posterior hypothalamus.

Stimulation of the anterior hypothalamus: Stimulation in the anterior hypothalamus, especially in the lateral part of it, brought about augmentation of the intrinsic wave. Usually this augmentation became gradually visible within 1-2 sec. after the start of the stimulation. With weak stimulation, the augmentation was confined to the ipsilateral olfactory bulb. If a stronger stimuls was used, the augmenting effect spread to the contralateral bulb. In the experiment

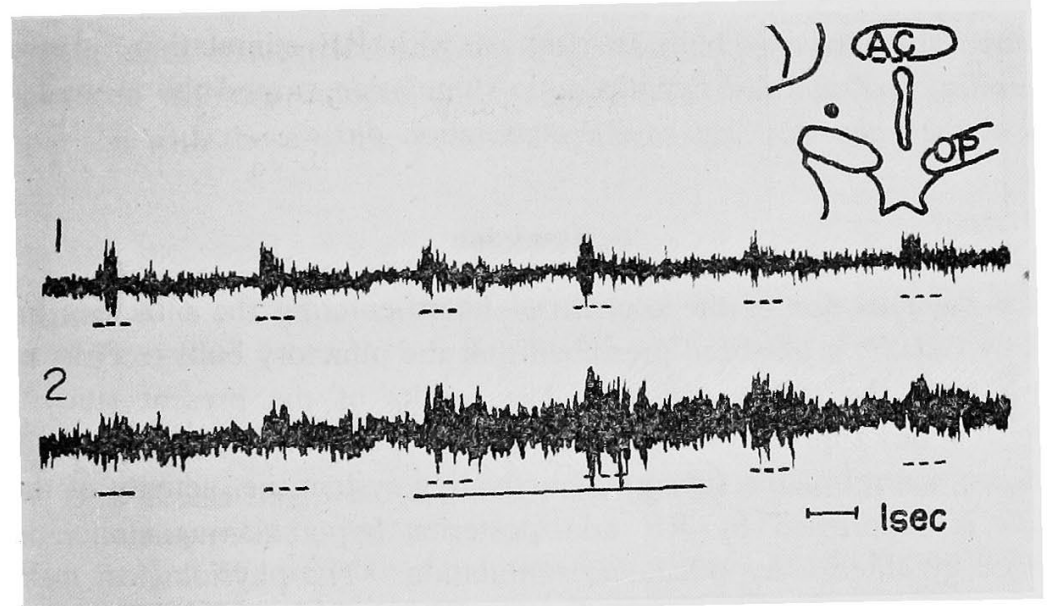

Fig. 2. Effects of anterior hypothalamic stimulation. Dotted lines indicate olfactory stimulus. 1 ; contralateral. 2 ; ipsilateral olfactory bulb. Intrinsic and induced waves were augmented in the ipsilateral olfactory bulb. Stimulus point was marked by a solid circle in the inset. AC; anterior commissure, OP; optic tract.

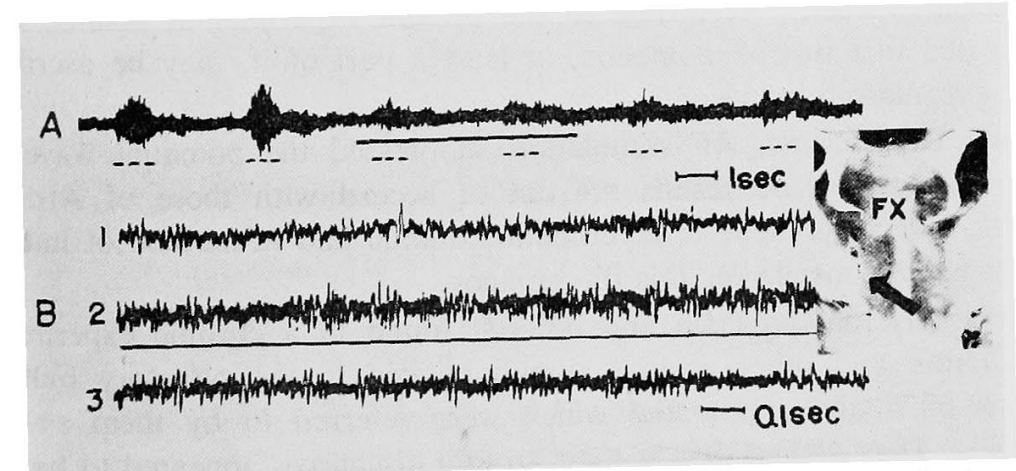

Fig. 3. Anterior hypothalamic stimulation. A; induced wave. B; intrinsic wave. Though intrinsic wave augmented through (2) and after (3) stimulation, induced wave was completely suppressed. Stimulus point was marked by arrow in the inset. FX; fornix. 
cited in Fig. 2, high frequency stimulation in the anterior hypothalamus augmented both the intrinsic and the induced waves in the ipsilateral bulb, having no remarkable effects upon the contralateral one. The induced wave, however, sometimes behaved contrary to the intrinsic wave. Fig. 3 mounts sample records showing the discrepancy between the intrinsic and the induced wave; the intrinsic wave increased in amplitude through and after stimulation of the anterior hypothalamus (B-2 and -3), whereas the same stimulus inhibited the induced wave (A). The differences in the condition of the experimental animal might be responsible for the discrepancy between the experimental results illustrated in Figs. 2 and 3. Thus, anterior hypothalamic stimulation had markedly different effects upon the olfactory bulb in contrast with RF stimulation. However, it was interesting that anterior hypothalamic stimulation caused the arousal reaction in EEG of the neocortex just as RF stimulation did.

\section{Discussion}

Since the presence of the centrifugal fibers entering the olfactory bulb was reported by $\mathrm{Caja}^{5}{ }^{5}$, it has been presumed that the olfactory bulb receives neuronal regulation from the higher centers. The results of the present study support unequivocally this supposition and show further that the olfactory bulb is under the duplicate control of the subcortical activating system, i.e. activity of the olfactory bulb is suppressed by RF and posterior hypothalamus stimulation and is enhanced by anterior hypothalamic stimulation. The physiological meaning of our findings will be the problem of a further investigation.

Though Kerr and Hagbarth ${ }^{3}$ ) found that high frequency stimulation of the tuberculum olfactorium and the periamygdaloid cortex augmented the induced wave of the ipsilateral olfactory bulb, they interpreted these results as merely due to antidromic stimulation of the fibers of the mitral cells coming via the lateral olfactory tract. According to the present experimental results, however, it is suggested that this phenomenon, at least a part of it, may be ascribed to a centrifugal regulatory influence.

In our experiments, RF stimulation suppressed the potential waves of the olfactory bulb and these results are out of accord with those of Arduini and Moruzzi ${ }^{2)}$. The differences in experimental animal and in method of immobilization might be responsible for this discrepancy.

Recently Hernández-Peón and others ${ }^{6}$ ) found in a chronic experiment that RF stimulation and other arousal stimuli elicited in the olfactory bulb of the cats bursts of rhythmic potential which were referred to by them as "asousal discharges". They argued that as their arousal discharges appeared to be different from the induced waves, this type of activity was brought about by centrifugal impulses. We, however, must accept their conclusion with reservation, because it seems to us that their arousal discharges are not a newly produced activity by 
centrifugal impulses, but simply represent the pre-existing induced waves which, however, are enhanced by augmented respiration due to general arousal reaction.

\section{Summary}

The functional relation between the olfactory bulb and the subcortical activating system was investigated in the curarized rabbits. The intrinsic and the induced waves of the olfactory bulb were suppressed by high frequency stimulation of the mesencephalic reticular formation and the posterior hypothalamus. In contrast to this, anterior hypothalamic stimulation mostly resulted in augmentation of both kinds of electrical activity, but in some cases only the intrinsic waves were enhanced with the induced ones suppressed. These findings were discussed in comparison with those of the previous investigators.

\section{References}

1) Livingston, R. B.: Central control of receptors and sensory transmission systems. Handbook of Physiology, Sec. 1, Vol. I, pp. 741-760. Am. Physiol. Soc., Washington, D. C., 1959.

2) Arduini, A. and Moruzzi, G.: Sensory and thalamic synchronization in the olfactory bulb. EEG Clin. Neurophysiol., 5, 235-242, 1953.

3) Kerr, D. I. B. and Hagbarth, K.-E.: An investigation of olfactory centrifugal fiber system. J. Neurophysiol., 18, 362-374, 1955.

4) Allison; A. C.: The structure of the olfactory bulb and its relationship to the olfactory pathways in the rabbits and the rat. J. Comp. Neur., 98, 309-353, 1953.

5) Ramón Y Cajal, S.: Histologie du Système Nerveux de l'Homme et des Vertébrés. Paris, Maloine, 1911.

6) Hernández-Peón, R., Lavín, A., Alcocer-Cuarón, C. and Marcelin, J. P.: Electrical activity of the olfactory bulb during wake-fulness and sleep. EEG Clin. Neurophysiol., $12,41-58,1960$. 